\title{
Parallel and antiparallel angular momentum transfer of circularly polarized light to photoelectrons and Auger electrons at the Ni $L_{3}$ absorption threshold
}

\author{
Fumihiko Matsui, ${ }^{*}$ Hiroshi Ota, and Kenji Sugita \\ Graduate School of Materials Science, Nara Institute of Science and Technology, 8916-5, Takayama, Ikoma, Nara 630-0192, Japan \\ Matthias Muntwiler \\ Paul Scherrer Institut, CH-5232 Villigen, Switzerland \\ Roland Stania and Thomas Greber \\ Physik Institut, Universität Zürich, CH-8057 Zürich, Switzerland
}

(Received 16 October 2017; published 18 January 2018)

\begin{abstract}
We report the investigation of the angular momentum transfer of light to photoelectrons and Auger electrons at the $\mathrm{Ni} L_{3}$ absorption threshold. Upon core-level excitation by circularly polarized light, the angular momentum of light, or helicity, is transferred to the emitted photoelectron. The angular momentum of the emitted photoelectron $\left(m_{\mathrm{f}}\right)$ is the sum of the helicity $(\sigma)$ and the orbital magnetic quantum number of the initial state $\left(m_{\mathrm{i}}\right)$. Here the quantization axis was defined as the direction along the incident light. This can be measured by the parallax shift of the forward focusing peak (FFP) direction in the photoelectron intensity angular distribution. At the absorption threshold, the excited core-level electron is promoted to a conduction-band state and the angular momentum of the light is partially transferred to Auger electrons. We measured photoelectron and Auger electron intensity angular distributions from the $\mathrm{Ni}(111)$ surface at the $L_{3}$ absorption threshold. We observed a significant angular circular dichroism of the [101] FFP for the $L_{3} M_{4,5} M_{4,5}$ Auger electrons. Furthermore, we discovered non-negligible reversal angular circular dichroism contrasts for the triplet components in the case of the $L_{3} M_{2,3} M_{4,5}$ Auger electrons, suggesting that the angular momentum of light was transferred to the emitted electron in the antiparallel way.
\end{abstract}

DOI: 10.1103/PhysRevB.97.035424

\section{INTRODUCTION}

Core-level photoelectron diffraction is a powerful technique to analyze element specific local atomic structures [1]. Forward focusing peaks (FFPs) appearing in the photoelectron intensity angular distribution indicate the directions of atoms surrounding a photoelectron emitter atom [2-5]. When a core level is excited by circularly polarized light (CPL), angular momentum of light, or helicity, is transferred to the emitted photoelectron, which can be confirmed by taking a stereograph of the atomic arrangement and measuring the parallax shift of the FFP directions induced by left and right CPL [6]. The parallax shift of the FFP is proportional to the angular momentum of the emitted photoelectron and inversely proportional to the interatomic distance between the photoelectron emitter and scattering atoms. The angular momentum of the emitted photoelectron $\left(m_{\mathrm{f}}\right)$ is the sum of the helicity $(\sigma)$ and the orbital magnetic quantum number (MQN) of the initial state $\left(m_{\mathrm{i}}\right)$. Angular circular dichroism (ACD) contrasts originate from the interference of the direct wave from the emitter atom and the wave scattered by the neighbor atoms. Thus they reflect the local atomic and electronic structure. This ACD contrast can be used to specify the emitter sites and investigate their atomic orbital characters [7].

*matui@ms.naist.jp
Compared to the photoelectron emission [8-10], the transition matrix element for the Auger electron emission, where several electronic states are involved, is much more complicated [11-13]. The radial factors of the transition matrix elements [14-16] for the different term final states have been studied for a long time but less attention was paid to the intensity angular distribution. There are theoretical simulations of Auger electron intensity angular distributions for low kinetic energy below $200 \mathrm{eV}$ by taking the angular momenta of the emitted electron waves into account $[17,18]$. Matsushita et al. demonstrated a holographic reconstruction of atomic arrangement from $\mathrm{Cu} L M M$ Auger electron diffraction by considering the Auger electron angular momentum [19]. The emitted Auger electron wave from an atom is not isotropic and contains the atomic orbital information of involved core and valence states.

Recently, a prominent FFP ACD was also found in the Auger electron intensity angular distribution at the absorption threshold [20-22]. Extraordinary large angular momentum transfer to the resonant Auger electron where the excited core electron is trapped at conduction-band state was found by Morscher et al. [20] for $\mathrm{Ni} L_{2}$ absorption and showed that the surface magnetization orientation can be determined in three dimensions. It is noteworthy that the ACD direction of $L_{3} M_{4,5} M_{4,5}$ was in opposite way with respect to that of $L_{2} M_{4,5} M_{4,5}$. We reported a quantitative analysis of angularmomentum-polarized $L M_{4,5} M_{4,5}$ Auger electrons from the 
nonmagnetic $\mathrm{Cu}$ surfaces at the $L_{3}$ and $L_{2}$ absorption thresholds and showed that the amount of angular momentum transfer depends on the term of the two-hole final state [21,22]. The amplitude and the direction of angular momentum transfer depend largely on the kinds of atomic orbitals involved in the Auger decay process. In the present study, we investigate the angular momenta of the $\mathrm{Ni} L_{3} M M$ Auger electrons experimentally. The resonant Auger electron emission by CPL excitation is an excellent way to specify the atomic number, orbital MQN, and atomic site of hole state in valence bands. This is useful for revealing the contribution of each atomic orbital to the electronic properties in compound crystals and epitaxial thin films.

\section{EXPERIMENTAL DETAILS}

The single-crystalline $\mathrm{Ni}(111)$ surface was sputtered with $\mathrm{Ar}^{+}$ions and annealed up to $500^{\circ} \mathrm{C}$ in ultrahigh vacuum condition to obtain a clean surface. The quality of the substrate surface was checked by electron diffraction, x-ray photoelectron spectroscopy, and $\mathrm{Ni}$ photoelectron and Auger electron angular distributions [20,23]. No contamination was identified. In order to seal the reactive $\mathrm{Ni}(111)$ surface [23], the surface was coated with a single layer of hexagonal boron nitride ( $h$-BN) $[24,25]$. Exchange splitting of the three $\bar{\Gamma}$ surface states of $\mathrm{Ni}(111)$ observed by three-dimensional spin- and angleresolved photoelectron spectroscopy, was also confirmed from the $h$-BN/Ni(111) surface. The $h$-BN layer does not affect the properties discussed here.

The photoelectron and Auger electron intensity angular distributions from the $h-\mathrm{BN} / \mathrm{Ni}(111)$ surface were measured using a concentric hemispherical energy analyzer at the soft $\mathrm{x}$ ray beamline X03DA [Photoemission and Atomic Resolution Laboratory (PEARL)] at the Swiss Light Source (SLS) [26,27]. The polarization of monochromatized soft $x$ rays at PEARL can be altered by changing the path of the storage ring electrons in the bending magnet section. The monochromator settings need not be changed for polarization switching. Ellipsoidalpolarized light with a polarization up to $70 \%$ was available. The emission angle at the center of the entrance slit was fixed at $60^{\circ}$ relative to the incident photon axis. The entrance slit of the analyzer was oriented vertically, such that the transition matrix elements for the linear polarization excitation become symmetric with respect to the detection center. The sample was mounted on a six-axis manipulator. All measurements were done at room temperature. The sample was not magnetized after annealing. All the spin information from various magnetic domains within the beam irradiated area was averaged.

\section{RESULTS AND DISCUSSION}

Figure 1 shows a resonant Ni $L_{3} M_{4,5} M_{4,5}$ Auger electron intensity angular distribution at the kinetic energy $E_{\mathrm{k}}$ of $840.5 \mathrm{eV}$ excited by linearly polarized light with photon energy $(h v)$ of $852.2 \mathrm{eV}$. The sample orientation relative to the analyzer direction, i.e., polar angle $\left(\theta_{\text {analyzer }}: 0^{\circ}-90^{\circ}\right.$, $1^{\circ}$ step $)$ and azimuthal angle $\left(\phi_{\text {analyzer }}:-165^{\circ}-165^{\circ}, 15^{\circ}\right.$ step $)$, were scanned. The transmission function of the analyzer for the intensity correction was obtained by averaging the data for 3185 scanned directions. The Auger electron intensity

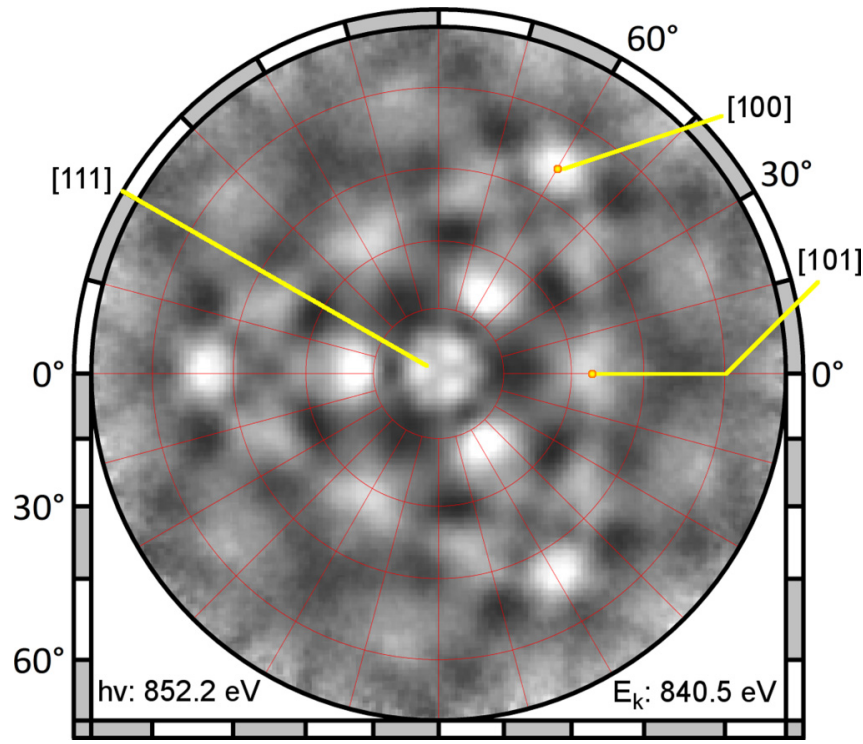

FIG. 1. A resonant Ni $L_{3} M_{4,5} M_{4,5}$ Auger electron intensity angular distribution from the $h$ - $\mathrm{BN} / \mathrm{Ni}(111)$ surface at the kinetic energy $\left(E_{\mathrm{k}}\right)$ of $840.5 \mathrm{eV}$ excited by linearly polarized light with photon energy $(h v)$ of $852.2 \mathrm{eV}$. Yellow lines indicate high-symmetry directions.

angular distribution pattern is plotted using the stereographic projection. The intense peak at the center corresponds to the FFP in the [111] direction. The [101] FFP corresponds to the direction of the nearest-neighbor atom. The interatomic distance $R_{[101]}$ is $0.249 \mathrm{~nm}$. Here, the in-plane azimuth $\phi$ was defined as the angle from the [112]] direction.

We measured the azimuthal angle dependence of this nearest-neighbor-atom FFP intensity at the [101] direction from the $\mathrm{Ni}(111)$ surface as a function of photoelectron kinetic energy; $I_{ \pm 1}\left(E_{\mathrm{k}}, \phi\right)$. CPL was used. The suffix denotes the helicity. Figure 2(a) shows the azimuthal-angle-integrated photoelectron spectra excited by CPL with photon energy of $852.2 \mathrm{eV}$ (spectrum A: black line) at $L_{3}$ absorption edge and $830 \mathrm{eV}$ (spectrum B: red line) well below absorption edge. Two spectra measured with the opposite helicity excitation $(\sigma=1$ and -1$)$ were added. The top abscissa indicates the kinetic energy for the spectrum A. The spectrum $\mathbf{B}$ having no Auger electron peak was shifted so that the positions of the Fermi level and each photoelectron peak match with those of the spectrum A. The $L_{3} M_{4,5} M_{4,5}$ Auger peak at $840.5 \mathrm{eV}$ in the spectrum $\mathbf{A}$ is a ${ }^{1} G$ two-hole final state corresponding to the resonance with the $3 d$ configuration interaction satellite. In the case of $L_{3} M_{2,3} M_{4,5}$ Auger electron peaks, the triplet and singlet components were observed. Powell and Mandl attributed these to the ${ }^{3} P$ and ${ }^{1} P$ two-hole final states [28]. The triplet has a higher kinetic energy due to the spin-orbit coupling.

Figure 2(b) shows the two-dimensional photoelectron intensity scan $I\left(E_{\mathrm{k}}, \phi\right)$ as functions of kinetic energy $E_{\mathrm{k}}$ and azimuthal angle $\phi$ near the [101] forward focusing direction. We averaged the two profiles excited by $\sigma= \pm 1$ helicities; $I\left(E_{\mathrm{k}}, \phi\right)=\left(I_{1}+I_{-1}\right) / 2$. Three intense vertical lines at the kinetic energy of 840.2, 776.0, and $769.0 \mathrm{eV}$ correspond to the different Auger electron peaks. 


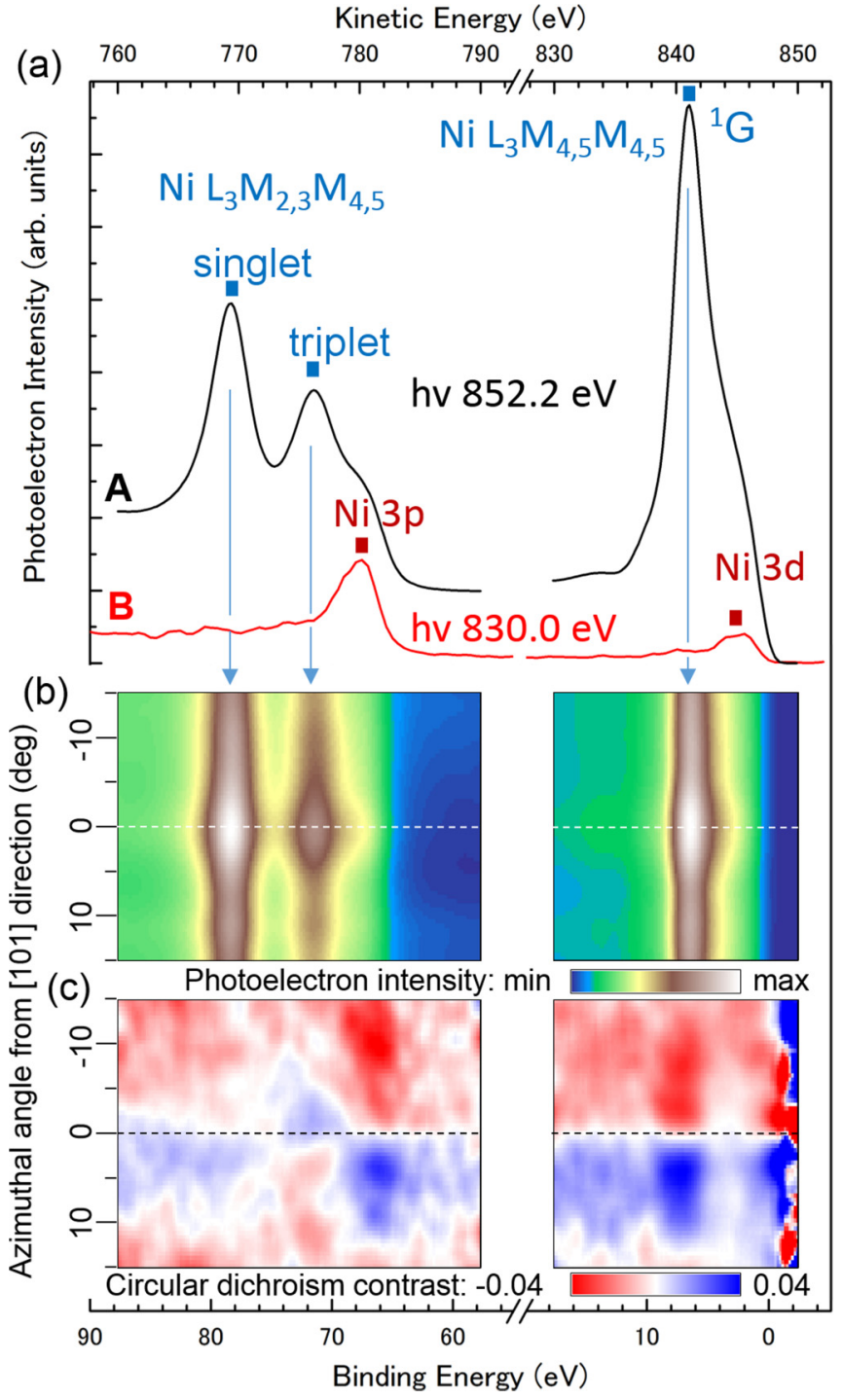

FIG. 2. (a) Photoelectron spectrum excited by $x$ rays with an energy of $852.2 \mathrm{eV}$ (spectrum A: black line) at the $L_{3}$ absorption edge and $830 \mathrm{eV}$ (spectrum B: red line) below the absorption edge. The top abscissa indicates the kinetic energy for spectrum $\mathbf{A}$, while spectrum $\mathbf{B}$ was shifted by $22.2 \mathrm{eV}$. (b) Angle-resolved photoelectron intensity and (c) circular dichroism contrast for spectrum A. The bottom abscissa indicates the binding energy for both spectra and panels.

Figure 2(c) shows the circular dichroism contrast of the scan region shown in Fig. 2(b). Circular dichroism contrast here is defined as $I_{\mathrm{CD}} \equiv\left(I_{1}-I_{-1}\right) /\left(I_{1}+I_{-1}\right)$. Offset intensities were subtracted as described in the Appendix. The prominent FFP ACD was observed in the $L_{3} M_{4,5} M_{4,5}$ Auger electron peak at the kinetic energy of $840.2 \mathrm{eV}$. The $3 p$ photoelectron FFP appeared at the kinetic energy of $780 \mathrm{eV}$, while the corresponding ACD contrast was maximum at $782 \mathrm{eV}$. The triplet $L_{3} M_{2,3} M_{4,5}$ Auger electron peak nearby seems to be canceling the lower kinetic energy part of the $3 p$ dichroism.

Figure 3 shows the azimuthal dependence of the five FFP intensity profiles for $3 d, L_{3} M_{4,5} M_{4,5}, 3 p$, triplet $L_{3} M_{2,3} M_{4,5}$, and singlet $L_{3} M_{2,3} M_{4,5}$. There exists non-negligible ACD contrast for the two $L_{3} M_{2,3} M_{4,5}$ Auger peaks. Note that the

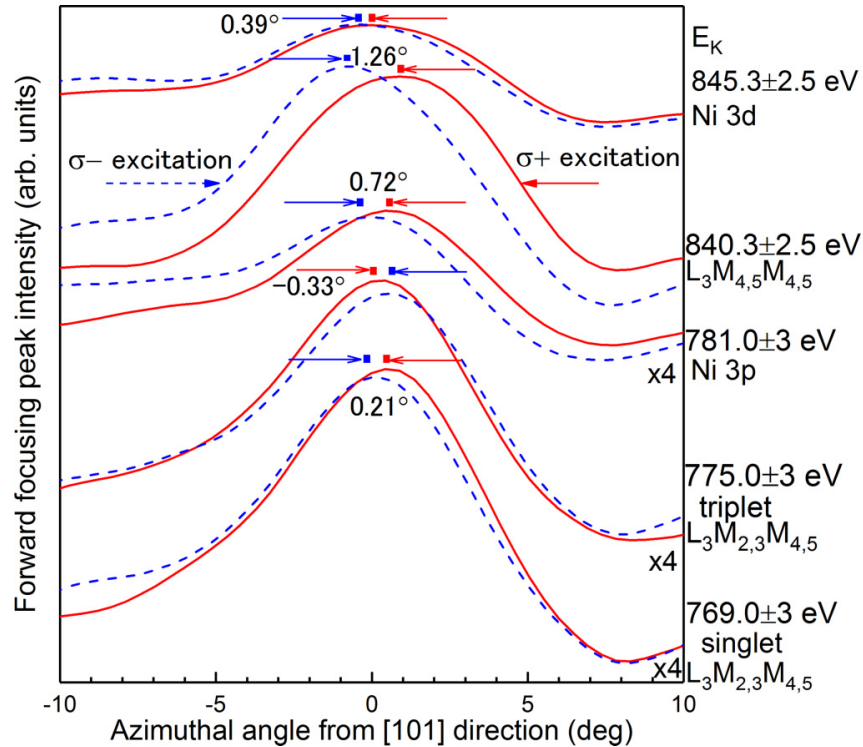

FIG. 3. Azimuthal profiles of the [101] FFP intensity for photoelectron and Auger electrons. Intensity within the indicated kinetic energy range is integrated for each azimuthal profile. Angular values indicate the difference of the two Gaussian peak positions fitted for each FFP.

ACD contrast for the triplet component is reversed in contrast to the other peaks.

The effective MQN of photoelectron, $m_{\mathrm{f}}^{*}(\theta)$, is the average of the different final-state angular momenta, $m_{\mathrm{f}}$, from the initial states of quantum number, $m_{\mathrm{i}}=m_{\mathrm{f}}-\sigma$, weighted by the transition probability at $\theta$, the polar angle from incident photon axis [29]. $\Theta_{l_{\mathrm{f}} m_{\mathrm{f}}}$ is a polar angle part of spherical harmonics. $m_{\mathrm{f}}^{*}(\theta)$ is as follows.

$$
m_{\mathrm{f}}^{*}(\theta)=\frac{\sum_{m_{\mathrm{i}}=-l_{\mathrm{i}}}^{l_{\mathrm{i}}} m_{\mathrm{f}}\left|c^{1}\left(l_{\mathrm{f}}, m_{\mathrm{f}}, l_{\mathrm{i}}, m_{\mathrm{i}}\right) \Theta_{l_{\mathrm{f}} m_{\mathrm{f}}}\right|^{2}}{\sum_{m_{\mathrm{i}}=-l_{\mathrm{i}}}^{l_{\mathrm{i}}}\left|c^{1}\left(l_{\mathrm{f}}, m_{\mathrm{f}}, l_{\mathrm{i}}, m_{\mathrm{i}}\right) \Theta_{l_{\mathrm{f}} m_{\mathrm{f}}}\right|^{2}} .
$$

The polar angle $(\theta)$ dependences of $p$ to $d$ and $d$ to $f$ transition probabilities were calculated and shown elsewhere [22]. For instance, in the case of $p \rightarrow d$ transition, the final states of $m_{\mathrm{f}}=0$ are the majority at the emission angle of $0^{\circ}$, while they are 0 at the magic angle of $54.7^{\circ}$. The final state $m_{\mathrm{f}}$ of 1 is the majority at $45^{\circ}$ and 0 at $0^{\circ}$ and $90^{\circ}$. The final state $m_{\mathrm{f}}$ of 2 is the majority at $90^{\circ}$, and 0 at $0^{\circ}$. In the present measurement geometry, $\theta$ is set to $60^{\circ}$, where the transition intensity ratio to the final states of $m_{\mathrm{f}}=0,1$, and 2 are 0.03:0.141:0.211.

The scattering intensity is described as the superposition of a reference wave and an object wave that leads to a diffraction pattern. The pronounced forward-scattering peaks emerge due to the phase shift in the non-point-like attractive potentials of the atoms that are probed by the emitted electrons. The forward-scattering direction is perpendicular to the wave front of the reference wave at the site of the scattering atom. The wave front of the reference wave is described with the photoelectron isophase plane $\exp \left[i\left(k r+m_{\mathrm{f}}^{*} \phi\right)\right]$, where $\phi$ is the azimuthal angle in the plane perpendicular to the incoming photon, and $m_{\mathrm{f}}^{*}$ is the effective magnetic quantum number of the emitted electron, which is nonzero in the case of the use of circularly polarized light, where the isophase plane is a 


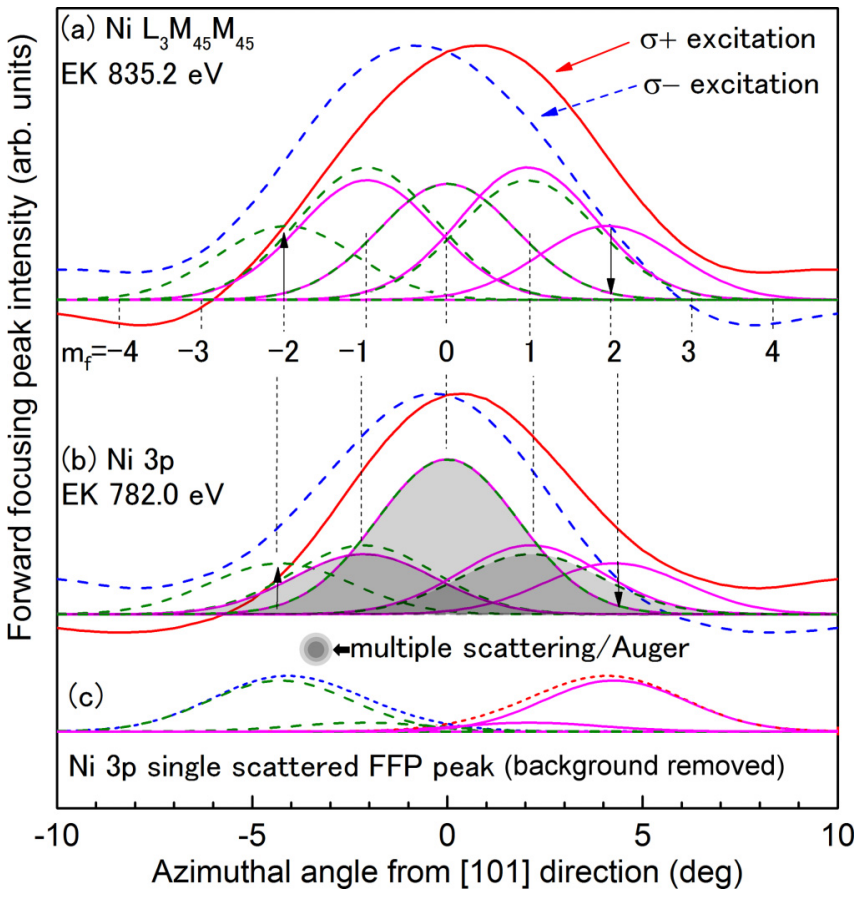

FIG. 4. Azimuthal profiles of the [101] FFP intensity for $\mathrm{Ni}$ (a) $L_{3} M_{4,5} M_{4,5}$ Auger electron and (b) $3 p$ photoelectron fitted with different angular momentum $\left(m_{\mathrm{f}}\right)$ components. Mirror symmetrization operation was applied. (c) Fitting of the spectra for (b). The multiple-scattering components are removed.

spiral [30]. This causes the rotation of the FFP direction around the incident light axis toward the same direction as the helicity of CPL. The rotation angle $\Delta \phi$ is well described by the Daimon formula $[6,30]$ :

$$
\Delta \phi=\tan ^{-1} \frac{m_{\mathrm{f}}^{*}(\theta)}{k R \sin ^{2} \theta},
$$

where $k$ is the wave number of the photoelectron. The shift $\Delta \phi$ is inversely proportional to the interatomic distance $R$ between the photoelectron emitting and the scattering atoms; in the present case, it is $R_{[101]}$. Thus, the local stereoscopic atomic arrangement can be imaged directly with a stereograph which consists of a pair of photoelectron intensity angular distributions excited by CPL [6,30-32] (not shown).

The magnitude of the FFP rotation upon electron excitation with CPL is also expected to decrease with multiple scattering since this weakens the influence of the phase of the reference wave. The azimuthal intensity profiles of the [101] FFPs excited by CPL were individually fitted by Gaussians corresponding to the different angular momenta $m_{\mathrm{f}}$ of emitted electrons and shown in Fig. 4. The Gaussian peaks are centered at $\phi=2.05^{\circ} m_{\mathrm{f}}$ and $2.13^{\circ} m_{\mathrm{f}}$ directions for $L_{3} M_{4,5} M_{4,5}$ and $3 p$, respectively. Solid and dashed lines correspond to the $\sigma=1$ and -1 excitation, respectively. Vertical arrows from the solid to dashed lines indicate the ACD contrasts.

The fitted result in Fig. 4(a) indicated that no sizable components of $m_{\mathrm{f}}= \pm 3$ and \pm 4 contribute to the FFP of $L_{3} M_{4,5} M_{4,5}$ Auger electron intensity angular distribution. The angular momenta range of $L M M$ Auger electrons is the same as that of $3 d$ electrons $\left(m_{\mathrm{i}}= \pm 2, \pm 1,0\right)$, which was also in

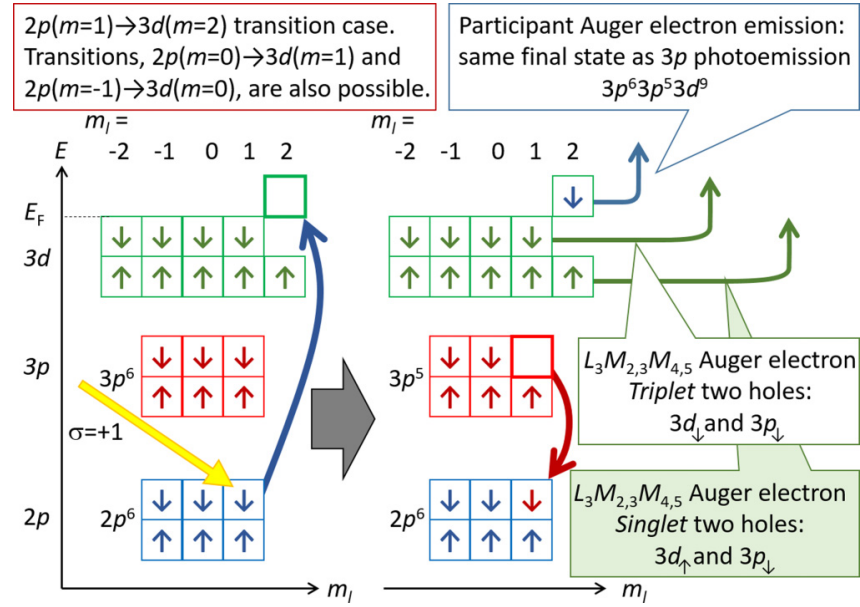

FIG. 5. Schematic diagram of an angular momentum transfer mechanism of x ray to emitted electrons for $L_{3} M_{2,3} M_{4,5}$.

the case of $\mathrm{Cu} L M M$ Auger electrons [33]. Therefore, the origin of ACD in the resonant Auger electron intensity angular distribution at the $L_{3}$ absorption threshold, where the excited core electron is trapped in the conduction band, is attributed to the final states $m_{\mathrm{f}}= \pm 2$.

In the case of $3 p$ core-level excitation, the photoelectrons gain angular momentum $\sigma$ by CPL excitation $\left(m_{\mathrm{f}}=2,1\right.$, and 0 ). The components for $m_{\mathrm{f}}=-1$ and -2 do not exist in the case of $3 p$ excitation by $\sigma=1$ helicity. Furthermore, the transition intensity of the final states of $m_{\mathrm{f}}=0$ is negligible since the emission angle $\theta=60^{\circ}$ is close to the node direction of $54.7^{\circ}$. Therefore, the fitted results for $m_{\mathrm{f}}=-1$ and 0 in the $\sigma=1$ excitation azimuthal angle profile are attributed to the multiple scattering and/or the contribution of adjacent $L_{3} M_{2,3} M_{4,5}$ Auger electrons. By this way, we evaluated the intensity which does not contribute to the ACD. They are indicated by the gray hatch in Fig. 4(b). This intensity was about $75 \%$ of the original FFP intensity. The intrinsic FFP intensity due to the interference of the direct and singlescattering wave at the nearest-neighbor atom is plotted in Fig. 4(c). For the precise evaluation of the multiple-scattering effect, the aid of theoretical simulation is inevitable. However, the measurement of the ACD contrast and its separation in the different $m_{\mathrm{f}}$ channels opens a way for quantifying the relative importance of multiple scattering with respect to single scattering.

Finally, we would like to give arguments on why antiparallel angular momentum transfer is observed for the triplet $L_{3} M_{2,3} M_{4,5}$ state. In the resonant $L_{3}$ absorption one $2 p$ electron is transferred to the remaining hole in the $\mathrm{Ni} 3 d$ shell. With the use of $\sigma=1$ light the $3 d m_{l}=2,1$, or 0 are populated from $2 p m_{l}=1,0$, or -1 . The direct Auger decay involving the $3 p m_{l}=1,0$, or -1 and the $3 d m_{l}=2$, 1 , or 0 states leads to the same final states as those of the direct photoemission from the $3 p$ state with the same photon energy. This emission has a parallel angular momentum transfer (see Fig. 3). The triplet Auger decay of the initially occupied $3 d$ states in turn, consists of the remaining $3 d$ electrons, which have a negative expectation value of $m_{l}$ and is therefore expected to produce an antiparallel momentum transfer as 
sketched in Fig. 5. The present model shown in Fig. 5 assumes a simple Hund's rule $3 d^{9}$ ground state despite crystal-field split and $3 d 4 s$ hybrids in the solid, and the antiparallel angular momentum transfer is qualitatively explained. In this picture we cannot, however, understand the apparent parallel angular momentum transfer in the singlet Auger decay that involves the decay of the majority $3 d$ electrons that have, for the case of $\mathrm{Ni}$, a full (isotropic) $3 d$ shell. The apparent dichroism of $0.21^{\circ}$ as reported in Fig. 3 might well be due to the dichroic background of the Ni $3 p$ photoemission that has a three times larger parallel angular momentum transfer $10 \mathrm{eV}$ above the singlet $L_{2} M_{2,3} M_{4,5}$ line. Such attenuation effects with the same order of magnitude have been observed for angular circular dichroism in germanium [34].

\section{CONCLUSIONS}

In conclusion, we have measured intensity angular distributions of resonant $L_{3} M_{4,5} M_{4,5}$ and $L_{3} M_{2,3} M_{4,5}$ Auger electrons excited by CPL, as well as that of $3 p$. In the case of $L_{3} M_{4,5} M_{4,5}$ Auger electrons where the excited core electron is trapped at the conduction band, the CDAD contrast was clearly observed. On the contrary, only small ACD contrast was observed for the $L_{3} M_{2,3} M_{4,5}$ Auger electron case. Interestingly, we discovered non-negligible ACD contrast and its inversed contrast for the singlet and triplet components of the $L_{3} M_{2,3} M_{4,5}$ Auger electrons, respectively, suggesting that the angular momentum transfer to the Auger electrons depends on the Auger decay path in a nontrivial way. An orbital MQN polarized state can be selected by ACD. Then, the character of the valence atomic orbital involved in the Auger electron ACD can be specified and the valence-band occupancy distribution for each orbital MNQ is evaluated.

\section{ACKNOWLEDGMENTS}

This work was performed with the approval of Paul Scherrer Institute. This research was supported by the Ministry of Education, Science, Sports and Culture, Grant-in-Aid for Scientific Research (B), 25287075, 15KK0167, 17H02911, and JSPS Grant-in-Aid for Scientific Research on Innovative Areas "3D Active-Site Science": 26105007 2604. The experiment at Switzerland was supported by Foundation for Nara Institute of Science and Technology.

\section{APPENDIX}

The procedure of data processing is described as the following. First, the set of two-dimensional photoelectron intensity

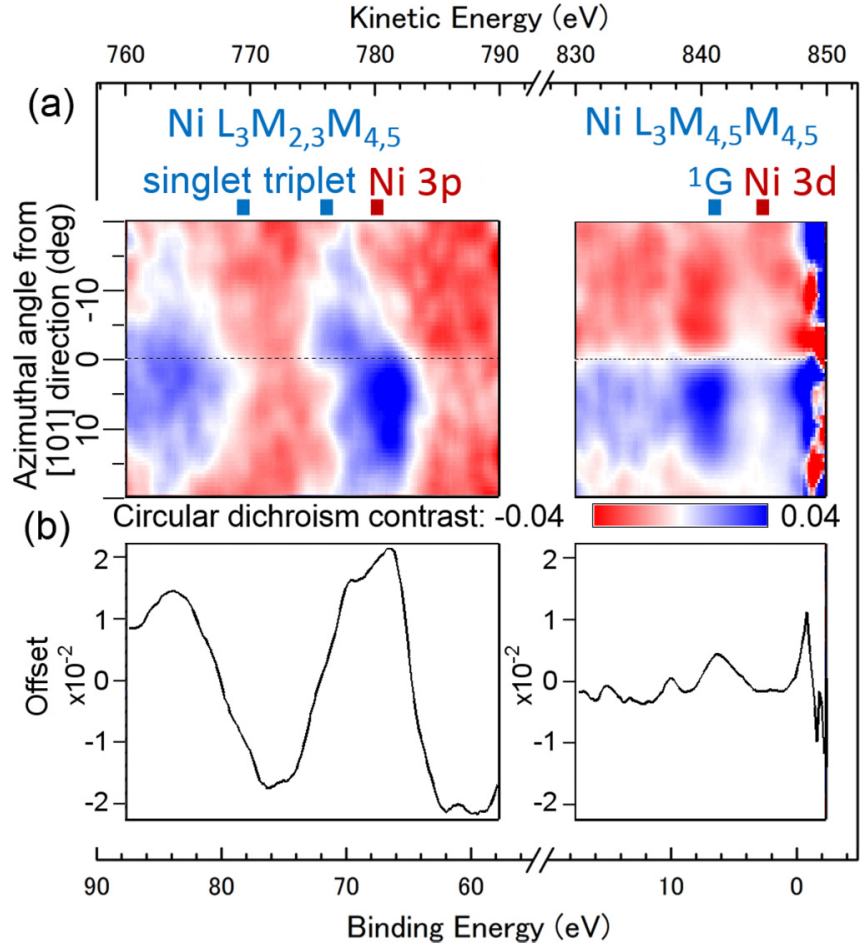

FIG. 6. (a) Circular dichroism contrast for the spectrum $\mathbf{A}$ shown in Fig. 2(a) before the offset correction. (b) Offset value for each kinetic energy obtained by averaging the circular dichroism over azimuthal angle. The top abscissa indicates the kinetic energy. The bottom abscissa indicates the binding energy for both spectra and panels.

scan $I_{ \pm 1}^{\text {raw }}\left(E_{\mathrm{k}}, \phi\right)$ measured was normalized by total intensity to correct the $\mathrm{x}$-ray intensity difference for $\sigma=1$ and -1 excitation. The intervals of scanned data points were $0.2 \mathrm{eV}$ and $0.25^{\circ}$. A Gaussian filter of 10 pixels was applied to reduce the noise. Then, circular dichroism contrast $I_{\mathrm{CD}}$ was calculated as shown in Fig. 6(a). We found the FFP position shift and its inversed one as shown in Fig. 3. However, such circular dichroism was not visible in the directly calculated $I_{\mathrm{CD}}$ pattern shown in Fig. 6(a). The offset deduced by averaging circular dichroism over azimuthal angle for each kinetic energy [Fig. 6(b)] was subtracted from the data shown in Fig. 6(a) and the result was presented as Fig. 2(c). The origin of the offset for $L_{3} M_{2,3} M_{4,5}$ is not clear at this stage.
[1] C. S. Fadley, J. Electron Spectrosc. Relat. Phenom. 178-179, 2 (2010).

[2] S. Kono, S. M. Goldberg, N. F. T. Hall, and C. S. Fadley, Phys. Rev. B 22, 6085 (1980).

[3] W. F. Egelhoff, Jr., Phys. Rev. B 30, 1052 (1984).

[4] H. C. Poon and S. Y. Tong, Phys. Rev. B 30, 6211 (1984).
[5] F. Matsui, T. Matsushita, Y. Kato, M. Hashimoto, K. Inaji, F. Z. Guo, and H. Daimon, Phys. Rev. Lett. 100, 207201 (2008).

[6] H. Daimon, Phys. Rev. Lett. 86, 2034 (2001).

[7] F. Matsui, T. Matsushita, Y. Kato, F. Z. Guo, and H. Daimon, J. Phys. Soc. Jpn. 76, 013705 (2007).

[8] S. M. Goldberg, C. S. Fadley, and S. Kono, J. Electron Spectrosc. Relat. Phenom. 21, 285 (1981). 
[9] T. Greber, J. Phys.: Condens. Matter 13, 10561 (2001).

[10] F. Matsui, H. Miyata, O. Rader, Y. Hamada, Y. Nakamura, K. Nakanishi, K. Ogawa, H. Namba, and H. Daimon, Phys. Rev. B 72, 195417 (2005).

[11] V. O. Kostroum, M. H. Chen, and B. Crasemann, Phys. Rev. A 3, 533 (1971).

[12] W. Bambynek, B. Crasemann, R. W. Fink, H.-U. Freund, H. Mark, C. D. Swift, R. E. Price, and P. Venugopara Rao, Rev. Mod. Phys. 44, 716 (1972).

[13] E. Antonides, E. C. Janse, and G. A. Sawatzky, Phys. Rev. B 15, 1669 (1977).

[14] P. J. Feibelman and E. J. McGuire, Phys. Rev. B 15, 3575 (1977).

[15] E. J. McGuire, Phys Rev. A 17, 182 (1978).

[16] S. Aksela and J. Sivonen, Phys. Rev. A 25, 1243 (1982).

[17] T. Greber, J. Osterwalder, S. Hüfner, and L. Schlapbach, Phys. Rev. B 45, 4540 (1992).

[18] T. Greber, J. Osterwalder, D. Naumović, A. Stuck, S. Hüfner, and L. Schlapbach, Phys. Rev. Lett. 69, 1947 (1992).

[19] T. Matsushita, F. Z. Guo, F. Matsui, Y. Kato, and H. Daimon, Phys. Rev. B 75, 085419 (2007).

[20] M. Morscher, F. Nolting, T. Brugger, and T. Greber, Phys. Rev. B 84, 140406(R) (2011).

[21] F. Matsui, M. Fujita, T. Ohta, N. Maejima, H. Matsui, H. Nishikawa, T. Matsushita, and H. Daimon, Phys. Rev. Lett. 114, 015501 (2015).

[22] F. Matsui, N. Maejima, H. Matsui, H. Nishikawa, H. Daimon, T. Matsushita, M. Muntwiler, R. Stania, and T. Greber, Z. Phys. Chem. 230, 519 (2016).
[23] T. Okuda, J. Lobo-Checa, W. Auwärter, M. Morscher, M. Hoesch, V. N. Petrov, M. Hengsberger, A. Tamai, A. Dolocan, C. Cirelli, M. Corso, M. Muntwiler, M. Klöckner, M. Roos, J. Osterwalder, and T. Greber, Phys. Rev. B 80, 180404(R) (2009).

[24] A. Nagashima, N. Tejima, Y. Gamou, T. Kawai, and C. Oshima, Phys. Rev. Lett. 75, 3918 (1995).

[25] W. Auwärter, T. J. Kreutz, T. Greber, and J. Osterwalder, Surf. Sci. 429, 229 (1999).

[26] P. Oberta, U. Flechsig, M. Muntwiler, and C. Quitmann, Nucl. Instrum. Methods Phys. Res., Sect. A 635, 116 (2011).

[27] M. Muntwiler, J. Zhang, R. Stania, F. Matsui, P. Oberta, U. Flechsig, L. Patthey, C. Quitmann, T. Glatzel, R. Widmer, E. Meyer, T. A. Jung, P. Aebi, R. Fasel, and T. Greber, J. Synchrotron Radiat. 24, 354 (2017).

[28] C. J. Powell and A. Mandl, Phys. Rev. Lett. 29, 1153 (1972).

[29] H. Daimon, S. Imada, and S. Suga, Surf. Sci. 471, 143 (2001).

[30] H. Daimon, T. Nakatani, S. Imada, S. Suga, Y. Kagoshima, and T. Miyahara, Jpn. J. Appl. Phys. 32, L1480 (1993).

[31] F. Matsui, T. Matsushita, and H. Daimon, J. Electron Spectrosc. Relat. Phenom. 178-179, 221 (2010).

[32] F. Matsui, N. Nishikayama, N. Maejima, H. Matsui, K. Goto, M. Hashimoto, T. Hatayama, T. Matsushita, Y. Kato, and H. Daimon, J. Phys. Soc. Jpn. 80, 013601 (2011).

[33] F. Matsui, T. Matsushita, and H. Daimon, J. Electron Spectrosc. Relat. Phenom. 195, 347 (2014).

[34] F. Matsui, M. Hashimoto, T. Matsushita, K. Goto, N. Maejima, H. Matsui, Y. Kato, and H. Daimon, J. Phys. Soc. Jpn. 81, 013601 (2012). 